\title{
Effect of autologous mesenchymal stem cells induced by low level laser therapy on cardiogenesis in the infarcted area following myocardial infarction in rats
}

\author{
Hana Tuby $^{1}$, Tali Yaakobi ${ }^{1}$, Lidya Maltz ${ }^{1}$, Yaakov Delarea ${ }^{2}$, Orit Sagi-Assif ${ }^{2}$, Uri Oron ${ }^{1 *}$ \\ ${ }^{1}$ Department of Zoology, The George S. Wise Faculty of Life Sciences, Tel-Aviv University, Tel-Aviv, Israel \\ ${ }^{2}$ Department of Cell Biology and Immunology, The George S. Wise Faculty of Life Sciences, Tel-Aviv University, Tel-Aviv, Israel \\ Email: "oronu@post.tau.ac.il
}

Received 27 May 2013; revised 29 June 2013; accepted 16 July 2013

Copyright (C) 2013 Hana Tuby et al. This is an open access article distributed under the Creative Commons Attribution License, which permits unrestricted use, distribution, and reproduction in any medium, provided the original work is properly cited.

\begin{abstract}
In this study, we investigated the hypothesis that photobiostimulation by low-energy laser therapy (LLLT) applied to the bone marrow (BM) of myocardial infarcted rats may attenuate the scarring processes that follow myocardial infarction (MI). Wistar rats underwent experimental MI. LLLT (Ga-Al-As diode laser) was applied to the BM of the exposed tibia at different time intervals post-MI ( 4 hrs, 48 hrs and 5 days). Sham-operated infarcted rats served as control. Infarct size was significantly reduced $(55 \%)$ in the laser-treated rats as compared to the control non-treated rats, at 2 weeks post-MI. A significant 3-fold increase was observed in the density of desmin immunopositive stained cells 14 days post-MI in the infarcted area of the laser-treated rats as compared to the non-laser-treated controls. The electron microscopy from the control infarcted rat hearts revealed a typical interphase area between the intact myocardium and the infarcted area, with conspicuous fibroblasts with collagen deposition dispersed among them. In rats that were laser treated (to $\mathrm{BM}$ ), the interphase zone demonstrated cells with different intracellular structures. There was also a significant increase in the percentage of c-kit positive cells and macrophages in the circulating blood of the laser treated rats as compared to control non treated ones. In the majority of the cells clusters of myofibrils anchored to well-developed Z-lines and structures resembling the morphological characteristics of mature intact cardiomyocytes were evident. In conclusion, LLLT to the BM of rats postMI induces cardiogenesis mainly at the borders of the infarcted area in the heart.
\end{abstract}

\footnotetext{
"Corresponding author.
}

Keywords: Low-Level Laser Therapy; Myocardial Infarction; Macrophage; Desmin; Ultrastructure; c-Kit Positive Cells

\section{INTRODUCTION}

Regenerative capacity and mitotic activity in the heart are confined mainly to the lower vertebrates [1]. Amputation of $\sim 20 \%$ of the zebrafish's ventricular myocardium resulted in full regeneration without scarring [2]. In amphibians, heart injury was associated with increased cell proliferation of myocytes and enhanced regeneration [3]. The adult mammalian heart was traditionally considered to be a post-mitotic organ with terminally differentiated cardiac myocytes. However, this dogma has recently been challenged by several studies and reviews [4-8]. These studies have suggested that cardiac myocytes are replaced throughout the lifespan even in the human heart, and that myocytes can regenerate from resident cardiac progenitor cells (CPC) as well as from bone marrow (BM). Studies in human infarcted hearts have shown evidence of cytokinesis of cells in the heart and evidence of cardiac stem cells that are activated in response to ischemic injury. This growth response is attenuated in chronic heart failure [9]. Some studies have reported that cardiac myocytes can be derived from BM; specifically, side population precursor cells following induction of myocardial infarction (MI) by left anterior descending artery (LAD) ligation [10-12]. Contradicting these findings, other laboratories using genetic markers have reported that lineage negative, c-kit+ BM cells did not differentiate into cardiomyocytes [13]. It was also suggested that BM-derived stem cells may stimulate the small population of stem cells in the ischemic heart to proliferate and differentiate to enhance cardiac repair post-MI [14]. In a recent study transient regenerative potential in the mouse heart was 
demonstrated during the neonatal period [15].

Low-level laser therapy (LLLT) has been found to modulate various biological processes [16,17], such as increasing mitochondrial respiration and ATP synthesis [18], facilitating wound healing and promoting the process of skeletal muscle regeneration and angiogenesis [1921]. In an experimental model of the infarcted heart in rats and dogs, it was demonstrated that LLLT application directly to the infarcted area in the heart at optimal power parameters significantly reduced scar tissue formation [22-24]. This phenomenon was partially attributed to a significant elevation in ATP content, heat shock proteins, vascular endothelial growth factor (VEGF), inducible nitric oxide (NO) synthase, and angiogenesis in the ischemic zone of the laser-irradiated rats, as compared to nonirradiated rats [25].

The effect of photobiostimulation on stem cells or progenitor cells has not been extensively studied. LLLT application to normal human neural progenitor cells significantly increases ATP production in these cells [26]. LLLT delivery to MSCs and cardiac stem cells in vitro caused a significant enhancement in their proliferation rate $[27,28]$. LLLT has also been shown to increase the proliferation rate of adipose-derived stem cells in vitro [29]. Recently, we demonstrated that LLLT application to autologous BM could induce mesenchymal stem cells (MSCs) in the BM to proliferate and cause their recruitment and specific homing in on the infarcted rat heart and not on other organs $[30,31]$. The laser treatment to the BM also caused a marked and statistically significant reduction of $79 \%$ in the scarring and ventricular dilatation followed MI as compared to infarcted non-lasertreated rats. The aim of the present study was to investigate the possibility that induction of stem cells in the BM of rats by LLLT could also affect cardiogenesis in the infarcted rat heart.

\section{MATERIALS AND METHODS}

\subsection{Experimental Procedures}

A total of 21 Wistar male rats, weighing $200-250$ gr, that underwent ligation of the LAD artery to induce MI, were used as described by us previously [23]. All the experimental procedures were approved by the animal care committee of Tel-Aviv University. Briefly, rats were anesthetized with Avertin ( $1 \mathrm{ml} / 100 \mathrm{~g}$ body weight I.P.) and the lungs were ventilated. Thoractomy was performed by invasion of the intercostals muscles between the $5^{\text {th }}$ and $6^{\text {th }}$ rib to expose the heart. The LAD artery was occluded $2 \mathrm{~mm}$ from the origin with 5-0 polypropylene thread (Ethicon Inc., Cincinnati, OH). Following LAD artery occlusion the chest muscles and skin were sutured and the rats were ventilated until they woke up. The infarcted rats were divided randomly into two groups. In one group LLLT was applied directly to the BM 4 hrs, 48 hrs and 5 days post-MI (see below). The second group was non-laser-treated (the rat's bone was exposed for the same duration as the laser-treated group but the laser was not turned on). Food and water were supplied ad libitum. Rats were sacrificed 14 days post-MI.

\subsection{Laser Application}

After induction of MI rats were randomly assigned to a laser-treated or control non-laser-treated group. A diode (Ga-Al-As) laser, wavelength $804 \mathrm{~nm}$ with a tunable power output of maximum of $400 \mathrm{~mW}$ (Lasotronic Inc., Zug, Switzerland) for application to the BM was used. The laser device was equipped with a metal-backed glass fiber optic (1.5 mm diameter). An infrared viewer (Lasotronic Inc. Zug, Switzerland) and infrared-sensitive detecting card (Newport, Inc., Irvine, CA) were used to determine the infrared irradiation area. Laser application was done by a $10 \mathrm{~mm}$ longitudinal cut in the skin above the medial aspect, and further delicate cleaning of the bone surface was carried out. The tip of the fiber optic (1.5 $\mathrm{mm}$ diameter) was placed perpendicularly to the center of the exposed medial aspect of the tibia and power density of $10 \mathrm{~mW} / \mathrm{cm}^{2}$ was applied to the BM. The laser was applied for a duration of $100 \mathrm{sec}$ (energy density 1.0 $\mathrm{J} / \mathrm{cm}^{2}$ ). Left or right exposed tibias were chosen at random for LLLT application. In sham-operated infarcted rats that served as control the tibias were exposed and the fiber optic was placed as described above but the laser beam was not turned on.

\subsection{Histology and Electron Microscopy}

A defined cross-section sample (2 $\mathrm{mm}$ thick) from the central part of the infarcted area was taken from all hearts for histology. Eight micron paraffin sections were prepared from the tissue samples of each heart. Infarct size was determined using Masson's trichrome staining as described by us previously [23]. Three observers, blinded to control or laser-treated rats, analyzed infarct size. Six microscopic slides from the infarcted area of each heart were chosen at random for determination of infarct size. Infarct size was expressed as the percentage of the total infarcted area relative to the total area of the left ventricle (LV) in each section, using image analysis software Sigma Scan Pro (Sigma, St. Louis, MO).

For electron microscopy three tissue samples from each of the control and laser-irradiated rat hearts were taken from the interphase zone between the infarcted and non-infarcted tissue by macroscopic examination. Fixation was performed in $3.5 \%$ glutaraldehyde in $0.1 \mathrm{M}$ cacodylate buffer for 24 hrs followed by embedment in Epon-812. Semi-thin sections (1 micron) were prepared in order to localize the interphase zone. Thin sections 
were then prepared and stained with uranyl acetate and lead citrate followed by examination with a Jeol electron microscope.

\subsection{Immunohistochemistry}

The total number of cells immunostained for desmin (bone marrow cells or newly formed) in the infarcted area were determined using a desmin kit (Zytomed Laboratory, Berlin, Germany). The procedure was performed at room temperature with anti-mouse (dilution 1:25 - 1:50) primary antibody for $60 \mathrm{~min}$. Following washing, slides were incubated with HRP secondary antibody for mouse for 30 min followed by DAB Chromogen system (Covance Inc., Dedham). Slides were rinsed again in wash buffer, stained in Hematoxylin for nuclei detection, mounted and viewed using a Zeiss microscope equipped with a camera and video screen. The total number of desmin immunostained cells within the infarcted area was counted and their density expressed as the percentage of the total area of the infarct using SigmaPro software.

\subsection{Flow Cytometry Analysis}

Blood samples were taken 2 and 7 days post-IR injury for fluorescence-activated cell sorting (FACS) analysis. $100 \mu \mathrm{l}$ of blood were mixed with different antibodies: anti-mouse CD117 (c-kit) PE (eBioscience San Diego, USA) and rat IgG2b isotype control PE (eBioscience San Diego, USA) and anti-rat macrophage marker PE (eBioscience San Diego, USA) and mouse IgG2a K isotype control PE (eBioscience San Diego, USA), were used for the FACS analysis according to the manufacturer's guidelines. Forty five min post incubation of the whole fresh blood with the relevant antibodies, $2 \mathrm{ml}$ of Fix/Lyse solution (eBioscience, San Diego, USA) was added. After mixture the suspended cells were left for $60 \mathrm{~min}$ in the dark at room temperature. Centrifugation was performed for $10 \mathrm{~min}$, supernatant was removed and washing of the pellet was performed with $2 \mathrm{ml}$ of Flow Cytometry Staining Buffer Solution (eBioscience, San Diego, California, USA). After another centrifugation for 10 minutes the supernatant was decanted. The pellet containing mononucleated cells was resuspended in $200 \mu$ l of flow stain buffer for FACS analysis.

\subsection{Statistical Analysis}

The SigmaStat 2.0 (Sigma, St. Luis, USA) software was used for statistical analysis. Tests were performed first for normality distribution, followed by parametric (student's t-test) test.

\section{RESULTS}

Application of LLLT to the infarcted heart caused a significant $(p=0.049)$ reduction of $55 \%$ in infarct size as compared to control. The present of macrophages and ckit positive cells in the blood was determined by FACS analysis (Figure 1). It was found that at 5 days post MI there was a statistical significant 2-fold higher concentration of macrophages and significant 1.4-fold higher c-kit positive cells (mesenchymal cells) in the laser treated rats as compared to the infarcted non laser treated rats. Desmin immunostaining of histological sections of the infarcted zone from laser-treated rats demonstrated a higher density of positively stained cells than in the non laser-treated ones (Figures 2-4). In the interphase zone, cells extending from the myocardium towards the in
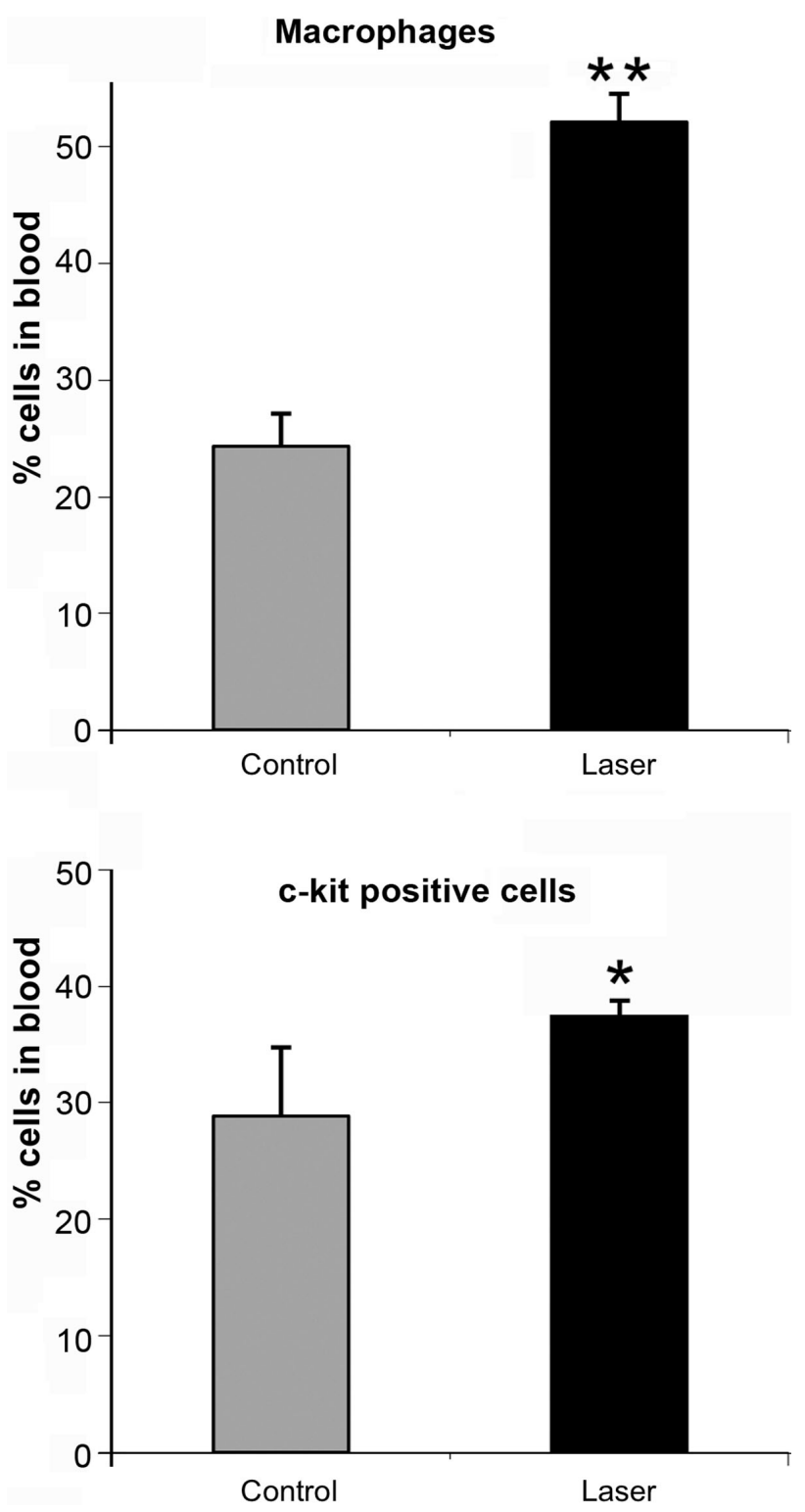

Figure 1. Percent (out of total mononucleated cells) of macrophages and c-kit positive cells in blood of control and laser treated rats (to the bone marrow) 5 days post MI as revealed by FACS analysis. The results are mean \pm S.E.M of 15 rats at each group. Statistical significance ${ }^{*} \mathrm{p}<0.05 ;{ }^{* *} \mathrm{p}<0.01$. 

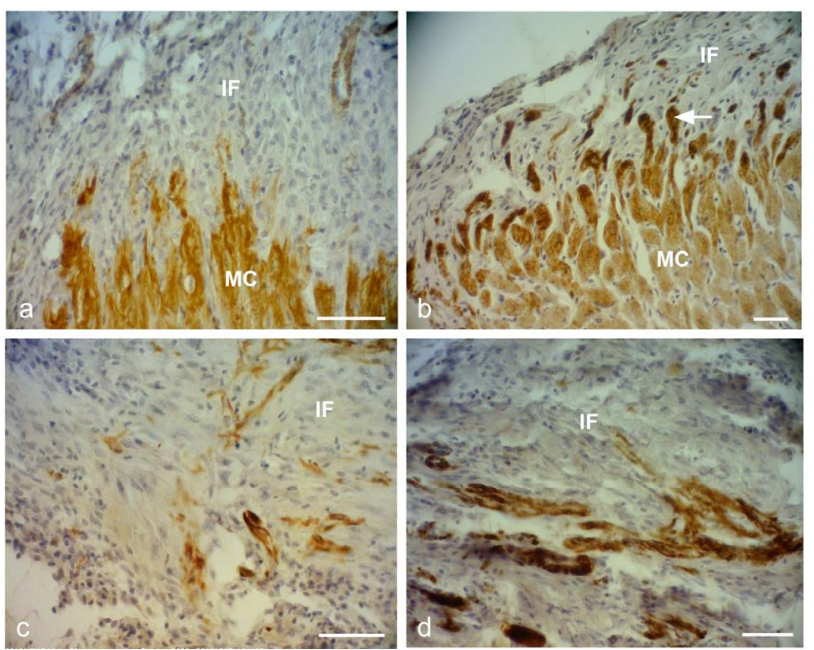

Figure 2. Representative desmin immunostained light micrographs of the infarcted zone of non-laser-treated rats (a, c) and laser-treated rats (to the bone marrow at 4 and $48 \mathrm{hrs}$ and 5 days) (b, d) taken 2 weeks post-MI. Note that the zone in the control non-laser-treated rats contains mainly collageneous material with a few desmin immunopositive cells in the infarcted area $(\mathrm{a}, \mathrm{c})$; while in the laser-treated rats the zone displays positive desmin staining in extended outgrowths (arrow) from the myocardium (MC) in (b), and in the cytoplasm of many cells in the infarcted area in (d). IF, Infarcted area. Bar $=50 \mu \mathrm{m}$.

farcted area showed higher immunostaining for desmin in the laser-treated rat hearts as compared to the control non-treated ones (Figure 2). The cell density of desmin immune-positive cells was also determined quantitatively in histological sections of both the infarcted laser-treated rats and infarcted non-laser-treated rats. The cell density was significantly $(\mathrm{p}<0.01) 3$-fold higher in the infarcted area of the laser-treated rats as compared to the non-laser-treated controls (Figure 4).

The electron micrographs of all samples taken from the control non-laser-treated infarcted rat hearts revealed a typical interphase area between intact and infarcted heart (Figure 5(a)). Adjacent to the non-ischemic intact myocardium there were conspicuous fibroblasts with collagen deposition dispersed among them (Figure 5(a)). In all samples taken from the laser-irradiated hearts the interphase zone between intact and infarcted area demonstrated different characteristics to those of the non-lasertreated infarcted rat hearts. Cells with newly-formed organized contractile myofilaments dispersed in the cytoplasm were detected in groups of several cells (Figure 5(b)). In these cells numerous mitochondria, clusters of ribosomes, and conspicuous clusters of contractile proteins were evident in the cytoplasm (Figures 6-8). Some cells contained dispersed contractile myofilaments in the cytoplasm that were still in an early stage of organization (Figure 6). The organization of newly-formed contractile myofilaments in the cytoplasm was observed in various
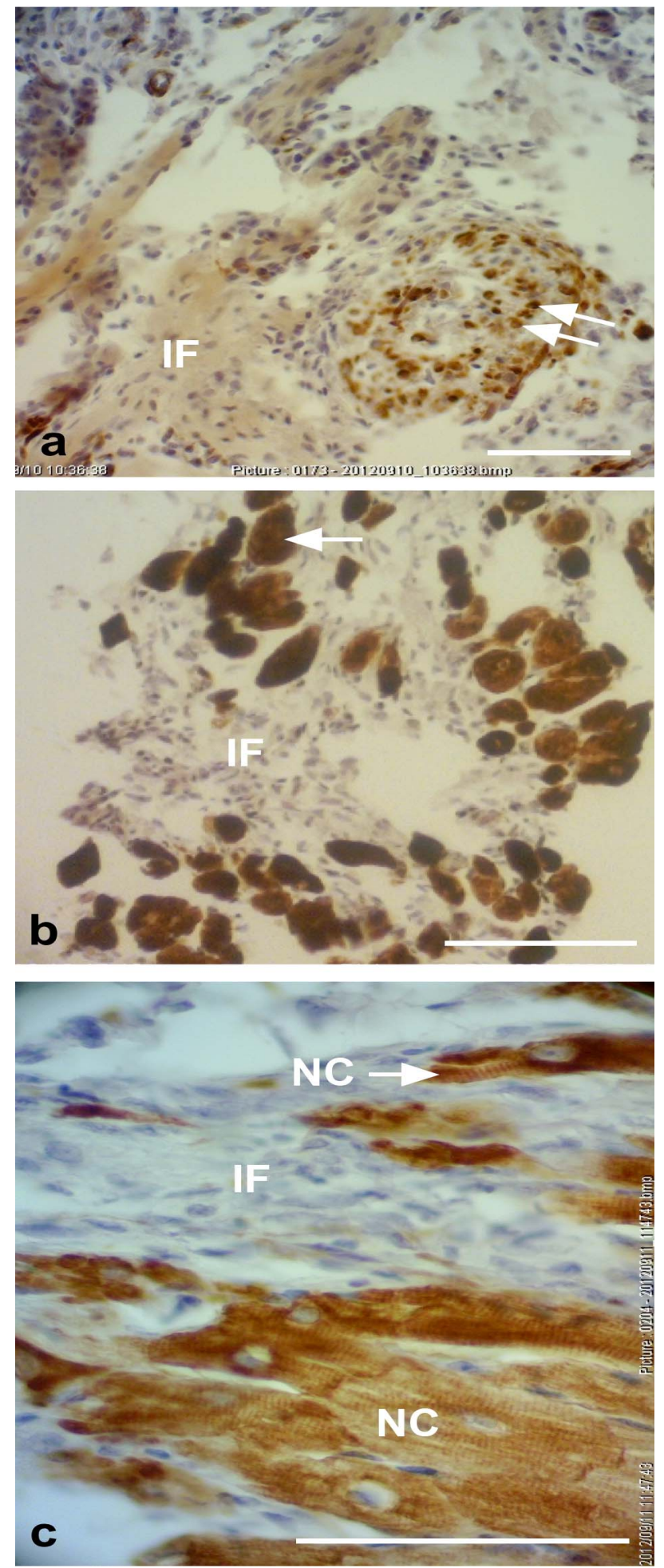

Figure 3. Representative desmin immunostained light micrographs of the interphase of the infarcted zone of laser-treated rats. Note that desmin positively stained cross-sections of myofibers (arrows) intermingled in the infarcted zone in (a). In (b) immunopositively stained cross-sections of myofibers (arrow) are visible in the infarcted area (IF). In (c) newly-formed cardiomyocytes (NC) are seen, with the desmin immunostaining mainly confined to the Z-line. Bar $=50 \mu \mathrm{m}$. 


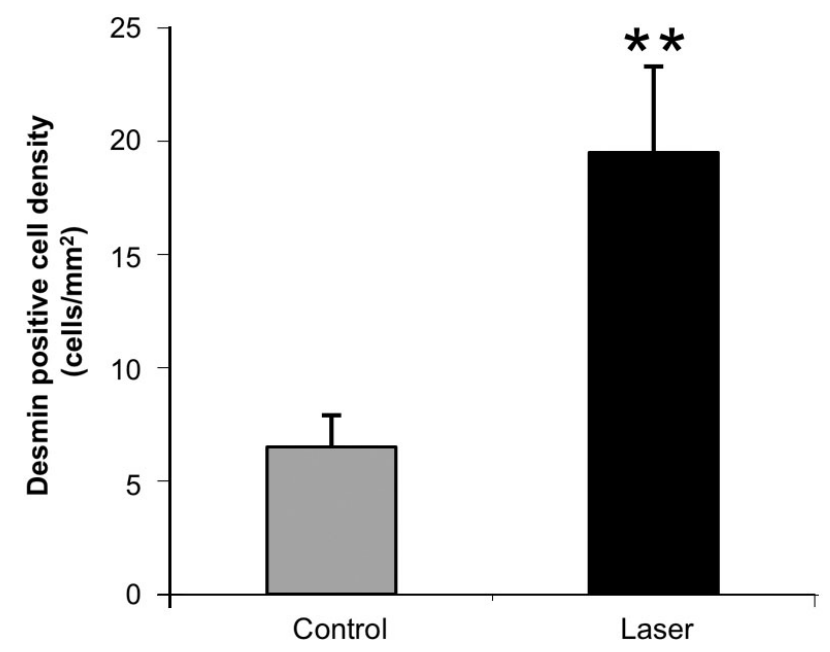

Figure 4. Density of desmin positively stained area (relative to total area) in the infarcted areas of control (non-laser-treated) and laser-treated (to the bone marrow) rats at 14 days post-MI. Results are mean+ S.E.M from 6 - 8 rats in each group. ${ }^{* *} \mathrm{p}<$ 0.01 .
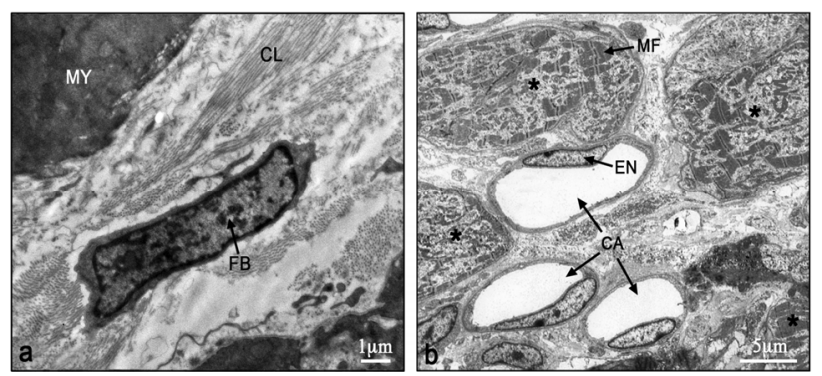

Figure 5. Electron micrographs of typical interphase zone between myocardium and infarcted area of control non-lasertreated (a) and laser-treated (b) to bone marrow rats. Note intact myocardium (MY) and adjacent fibroblast (FB) in the infarcted area surrounded by collagen (CL) deposition in (a). In (b) several newly-formed cardiomyocytes (marked with asterix) with conspicuous well-organized myofilaments (MF) in their cytoplasm are evident adjacent to blood capillaries (CA). EN, Endothelial cell.

degrees of maturation in those cells. In some cells the myofilaments were dispersed in the cytoplasm and in others they were organized in clusters anchored to welldeveloped Z-lines (Figure 7(a)). In certain cells the myofilaments were organized parallel to the longitudinal direction of the cells, resembling the morphological characteristics of mature intact cardiomyocytes (Figure 7(b)). Some of the cells were also seen in a process of formation of typical intercalated disc between them (Figure 9).

\section{DISCUSSION AND CONCLUSION}

The most significant outcome of this study was the appearance of newly-formed cardiomyocytes following laser treatment to the BM, as indicated by light and electron microscopy. There was a 3-fold increase in the density of

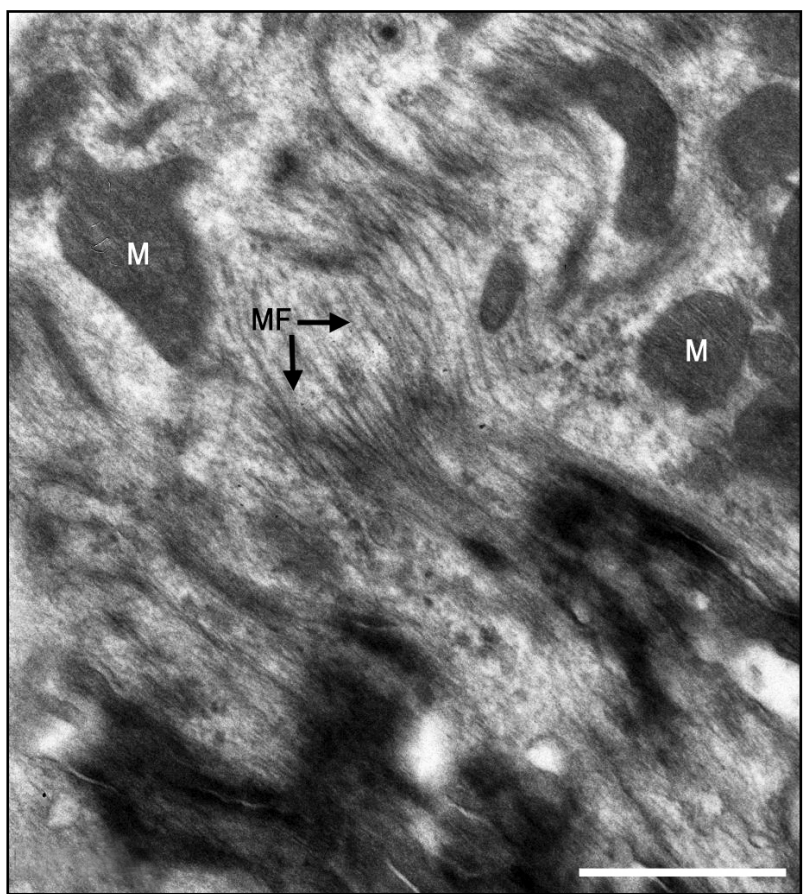

Figure 6. Electron micrographs of most probably newly-formed cardiomyocytes at an early stage of organization of contractile myofilaments. Note myofilaments (MF) in the cytoplasm. M, Mitochondrion. Bar $=1 \mu \mathrm{m}$.
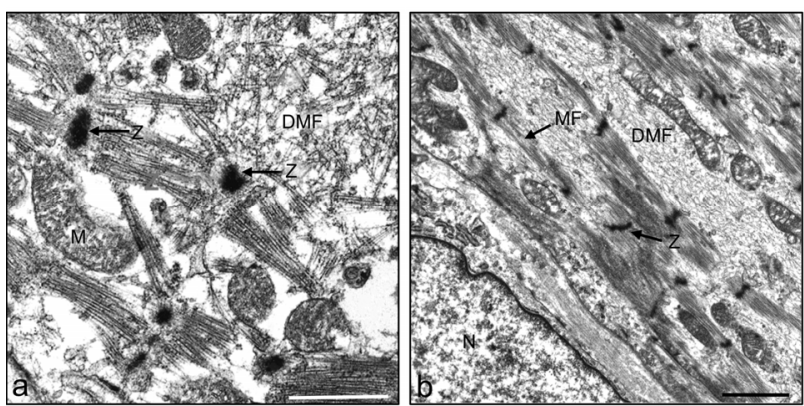

Figure 7. Electron micrographs of most probably newly-formed cardiomyocytes with early (a) and late (b) stages of the organization of the contractile myofilaments in the cytoplasm. Note contractile myofilaments that are dispersed (DMF) in the cytoplasm with a few organized in clusters anchored to Z-lines (Z) in (a). In (b) myofilaments (MF) are organized in parallel to the longitudinal axis of the cardiomyocyte, resembling their organization in mature cardiomyocyte. N, Nucleus. Bar $=1 \mu \mathrm{m}$.

desmin immunostained cells in the infarcted rat hearts that had been laser treated. Desmin is a protein found in the cytoplasm of developing myocytes and cardiomyocytes [32]. The significantly higher occurrence of desmin-positive cells in the infarcted area of the lasertreated hearts may indicate the synthesis of new contractile proteins in the developing new cardiomyocytes, resembling the process that takes place during embryonic development. The ultrastructural features of the cells in the interphase between the intact myocardium and the 

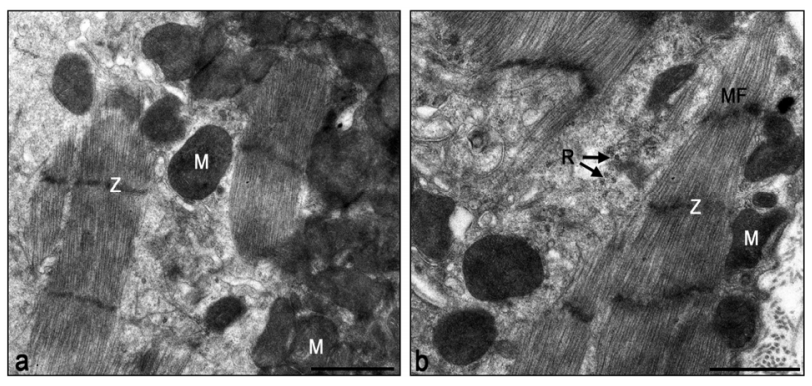

Figure 8. Electron micrographs of typical interphase zone between myocardium and infarcted area of laser-treated infarcted rat heart. Note numerous mitochondria $(\mathrm{M})$ in the cytoplasm of the cardiomyocytes in (a) and (b). Also note organized contractile myofilament with well-developed Z-lines $(\mathrm{Z})$, some dispersed myofilaments and clusters of ribosomes $(\mathrm{R})$. Bar $=1$ $\mu \mathrm{m}$.

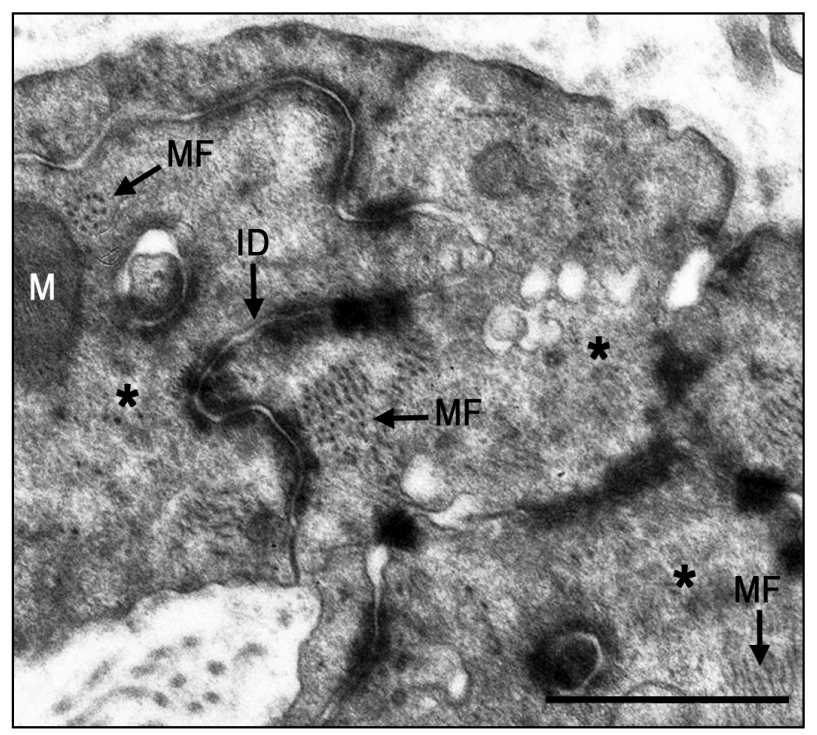

Figure 9. Electron micrographs of typical intercalated disk formation in the interphase region of the infarcted heart of laser-treated rats. Formation of intercalated disks (ID) between cells (marked with asterix) is evident. Note that the most probably newly-formed cardiomyocytes contain clusters of myofilaments (MF) in the cytoplasm that are conspicuous in their oblique or cross-sections (arrows). Bar $=1 \mu \mathrm{m}$.

infarcted myocardium of the laser-treated rats, as shown in this study, clearly resemble the characteristics of cardiomyocytes during embryonic development of the heart [33]. Furthermore, the clusters of ribosomes and the numerous clusters of mitochondria in the cytoplasm of these cells may characterize cells that are active in the synthesis of proteins. It was previously demonstrated that direct LLLT to the infarcted hearts of rats, dogs and pigs caused a significant reduction of scarring post-MI [23,24]. It was suggested that part of this reduction could be explained by the regenerative response that takes place in the interphase zone [24].

The results of the present study indicate that the LLLT applied to autologous BM attenuates the concentration of macrophages and MSC in the circulating blood. We have previously shown that LLLT application to the BM of infarcted rats caused a 2 fold enhancement in the rate of proliferation of MSC in the BM [30]. Those cells that most probably leave the BM to the circulating blood indeed show a significant elevation of their concentration (as reveled by the FACS analysis in the present paper) at 5 days post MI. Consequently these cells probably home in on the infarcted heart, and even migrate specifically to the infarcted area [30]. These cells may induce cardiac stem cells to differentiate to newly-formed cardiomyocytes, as suggested previously by Hatzistergos et al. [14]. Indeed, it was found that endogenous c-kit+ cardiac stem cells were increased by 20 -fold in the rat infarcted heart compared to control, following transcardial injection of BM-derived MSCs [14]. Such induction may be enabled due to paracrine secretion of various growth factors by the laser-stimulated MSC that originated from the BM. The possibility that paracrine secretion occurs in implanted stem cells during cell therapy to the heart postMI has been suggested previously [34]. Another mechanism that may take place after homing of stem cells to the infarcted heart of the laser-stimulated rats is that these cells continue to proliferate in the appropriate milieu of the interphase zone in the infarcted heart and then differentiate to cardiomyocytes [30].

Another possible mechanism that maybe associates with the reduction of infarct size is the significant increase in the concentration of macrophages in the circulation following LLLT to the BM as revealed from the FACS analysis in the present study. These findings corroborate with studies indicating that macrophages activity in the infarcted area at early stages post MI cause reduction of scarring post MI $[35,36]$. Thus, it could be postulated that more macrophages that will eventually home in the infarcted area from the circulating blood in the laser treated rats will also contribute to the reduction of scarring.

Although the findings of the present study do not indicate the extent of regenerative capacity of the rat infarcted heart post-laser-irradiation, they do reveal a shift from practically no cardiomyocytes in the tissue samples taken from the non-laser-treated hearts, to the presence of newly-formed cardiomyocytes in all the electron microscope sections taken from the hearts of rats that are laser-treated to the BM.

In conclusion, to the best of our knowledge, this is the first study to demonstrate the appearance of newly-formed cardiomyocytes in the infarcted area following LLLT to autologous BM in the infarcted rat heart. The mechanisms associated with this phenomenon remain to be elucidated in further studies. 


\section{ACKNOWLEDGEMENTS}

This study was partially supported by the Elizabeth and Nicholas Shlezak Super-center for Cardiac Research and Medical Engineering. The authors wish to acknowledge N. Paz for editing the manuscript and V. Wexler for helping with preparation of the figures.

\section{REFERENCES}

[1] Rumyantsev, P.P. (1977) Interrelations of the proliferation and differentiation processes during cardiac myogenesis and regeneration. International Review of Cytology, 51, 186-273. doi:10.1016/S0074-7696(08)60228-4

[2] Poss, K.D., Wilson, L.G. and Keating, M.T. (2002) Heart regeneration in zebrafish. Science, 298, 2188-2190. doi:10.1126/science.1077857

[3] Rumyantsev, P.P. (1973) Post-injury DNA synthesis, mitosis and ultrastructural reorganization of adult frog cardiac myocytes. An electron microscopic-autoradiographic study. Z Zellforsch Mikrosk Anat, 139, 431-50. doi:10.1007/BF00306596

[4] Barnett, P. and van den Hoff, M.J.B. (2011) Cardiac regeneration: Different cells same goal. Medical \& Biological Engineering \& Computing, 49, 723-732. doi:10.1007/s11517-011-0776-5

[5] Bollini, S., Smart. N. and Riley, P.R. (2011) Resident cardiac progenitor cells: At the heart of regeneration. Journal of Molecular and Cellular Cardiology, 50, 296-303. doi:10.1016/j.yjmcc.2010.07.006

[6] Choi, W.Y. and Poss, K.D. (2012) Cardiac regeneration. Current Topics in Developmental Biology, 100, 319-343. doi:10.1016/B978-0-12-387786-4.00010-5

[7] Laflamme, M.A. and Murry, C.E. (2011) Heart regeneration. Nature, 473, 326-335. doi:10.1038/nature10147

[8] Steinhauser, M.L. and Lee, R.T. (2011) Regeneration of the heart. EMBO Molecular Medicine, 3, 701-712. doi:10.1002/emmm.201100175

[9] Urbanek, K., Torella, D., Sheikh, F., De Angelis, A., Nurzynska, D., Silvestri, F., Beltrami, C.A., Bussani, R., Beltrami, A.P., Quaini, F., Bolli, R., Leri, A., Kajstura. J. and Anversa, P. (2005) Myocardial regeneration by activation of multipotent cardiac stem cells in ischemic heart failure. Proceedings of the National Academy of Sciences of the USA, 102, 8692-8697. doi:10.1073/pnas.0500169102

[10] Bittner, R.E., Schofer, C., Weipoltshammer, K., Ivanova, S., Streubel, B., Hauser, E., Freilinger, M., Höger, H., ElbeBürger, A. and Wachtler, F. (1999) Recruitment of bonemarrow-derived cells by skeletal and cardiac muscle in adult dystrophic mdx mice. Anatomy and Embryology, 199, 391-396. doi:10.1007/s004290050237

[11] Jackson, K.A., Majka, S.M., Wand, H., Pocius, J., Hartley, C.J., Majesky, M.W., Entman, M.L., Michael, L.H., Hirschi, K.K. and Goodell, M.A. (2001) Regeneration of ischemic cardiac muscle and vascular endothelium by adult stem cells. The Journal of Clinical Investigation, 107, 1395-1402. doi:10.1172/JCI12150

[12] Pfister, O., Mouquet, F., Jain, M., Summer, R., Helmes, M., Fine, A., Colucci, W.S. and Liao, R. (2005) CD3- but not CD31+ cardiac side population cells exhibit functional cardiomyogenic differentiation. Circulation Research, 97, 52-61. doi:10.1161/01.RES.0000173297.53793.fa

[13] Balsam, L.B., Wagers, A.J., Christensen, J.L., Kofidis, T., Weissman, I.L. and Robbins, R.C. (2004) Haematopoietic stem cells adopt mature haematopoietic fates in ischaemic myocardium. Nature, 428, 668-673. doi: $10.1038 /$ nature 02460

[14] Hatzistergos, K.E., Quevedo, H., Oskouei. B.N., Hu, Q., Feigenbaum, G.S., Margitich, I.S., Mazhari, R., Boyle, A.J., Zambrano, J.P., Rodriguez, J.E., Dulce, R., Pattany, P.M., Valdes, D., Revilla, C., Heldman, A.W., McNiece, I. and Hare, J.M. (2010) Bone marrow mesenchymal stem cells stimulate cardiac stem cell proliferation and differentiation. Circulation Research, 107, 913-922. doi:10.1161/CIRCRESAHA.110.222703

[15] Porrello, E.R., Mahmoud, A.I., Simpson, E., Hill, J.A., Richardson, J.A., Olson, E.N. and Sadek, H.A. (2011) Transient regenerative potential of the neonatal mouse heart. Science, 331, 1078-1080. doi:10.1126/science.1200708

[16] Conlan, M.J., Rapley, J.W. and Cobb, C.M. (1996) Biostimulation of wound healing by low energy laser irradiation. Journal of Clinical Periodontology, 23, 492-496. doi:10.1111/j.1600-051X.1996.tb00580.x

[17] Karu, T. (2007) Ten lectures on basic science of laser photherapy. Prima Books, Gragesberg.

[18] Karu, T. (2010) Mitochondrial mechanisms of photobiomodulation in context of new data about multiple roles of ATP. Photomedicine and Laser Surgery, 28, 159-160. doi:10.1089/pho.2010.2789

[19] Bibikova, A. and Oron, U. (1993) Promotion of muscle regeneration in the toad (Bufo viridis) gastrocnemius muscle by low energy laser irradiation. Anatomical Record, 235, 374-380. doi:10.1002/ar.1092350306

[20] Bibikova, A., Belkin, A. and Oron, U. (1994) Enhancement of angiogenesis in regenerating gastrocnemius muscle of the toad (Bufo viridis) by low energy laser irradiation. Anatomy and Embryology, 190, 597-602. doi:10.1007/BF00190110

[21] Oron, U. (2006) Photoengineering of tissue repair in skeletal and cardiac muscles. Photomedicine and Laser Surgery, 24, 111-120. doi:10.1089/pho.2006.24.111

[22] Yaakobi, T., Shoshani, Y., Levkovitz, S., Rubin, O., BenHaim, S.A. and Oron, U. (2001) Long term effect of low energy laser irradiation on infarction and reperfusion injury in the rat heart. Journal of Applied Physiology, 90, 2411-2441.

[23] Oron, U., Yaakobi, T., Oron, A., Hayam, G., Gepstein, L., Wolf, T., Rubin, O. and Ben Haim, S.A. (2001a) Attenuation of the formation of scar tissue in rats and dogs post myocardial infarction by low energy laser irradiation. $L a-$ sers in Surgery and Medicine, 28, 204-211. doi:10.1002/lsm.1039

[24] Oron, U., Yaakobi, T., Oron, A., Mordechovitz, D., Shofti, R., Hayam, G., Dror, U., Gepstein, L., Wolf, T., Haudenschild, C. and Ben Haim, S.A. (2001b) Low energy laser irradiation reduces formation of scar tissue following myocardial infarction in dogs. Circulation, 103, 296-301. 


\section{doi:10.1161/01.CIR.103.2.296}

[25] Tuby, H., Maltz, L. and Oron, U. (2006) Modulations of VEGF and iNOS in the rat heart by low energy laser irradiation are associated with cardioprotection and enhanced angiogenesis. Lasers in Surgery and Medicine, 38, 682688. doi:10.1002/lsm.20377

[26] Oron, U., Ilic, S., De Taboada, L. and Streeter, J. (2007) Ga-As (808 nm) laser irradiation enhance ATP production in human neuronal cells in culture. Photomedicine and Laser Surgery, 25, 180-182. doi:10.1089/pho.2007.2064

[27] Tuby, H., Maltz, L. and Oron, U. (2007) Low-level laser irradiation (LLLI) promotes proliferation of mesenchymal and cardiac stem cells in culture. Lasers in Surgery and Medicine, 39, 373-378. doi:10.1002/lsm.20492

[28] Li, W.T., Leu, Y.C. and Wu, J.L. (2010) Red-light lightemitting diode irradiation increases the proliferation and osteogenic differentiation of rat bone marrow mesenchymal stem cells. Photomedicine and Laser Surgery, 28, S-157-S-165. doi:10.1089/pho.2009.2540

[29] Mvula, B., Moore, T.J. and Abrahamse, H. (2010) Effect of low-level laser irradiation and epidermal growth factor on adult human adipose-derived stem cells. Lasers in Medical Science, 25, 33-39. doi:10.1007/s10103-008-0636-1

[30] Tuby, H., Maltz, L. and Oron, U. (2011) Induction of autologous mesenchymal stem cells in the bone marrow by low-level laser therapy has profound beneficial effects on the infarcted rat heart. Lasers in Surgery and Medicine,
43, 401-409. doi:10.1002/lsm.21063

[31] Oron, U. (2011) Light therapy and stem cells: A therapeutic intervention of the future. Journal of Interventional Cardiology, 3, 627-629.

[32] Toma, C., Pittenger, M.F., Cahill, K.S., Byrne, B.J. and Kessler, P.D. (2002) Human mesenchymal stem cells differentiate to a cardiomyocyte phenotype in the adult murine heart. Circulation, 105, 93-98. doi:10.1161/hc0102.101442

[33] Oron, U. and Mandelberg, M. (1985) Focal regeneration in the rat myocardium following cold injury. Cell Tissue Research, 241, 459-463. doi:10.1007/BF00217194

[34] Mummery, C.L., Davis, R.P. and Krieger, J.E. (2010) Challenges in using stem cells for cardiac repair. Science Translational Medicine, 14, 1-5.

[35] van Amerongen, M.J., Harmsen, M.C., van Rooijen, N., Petersen, A.H. and van Luyn, M.J. (2007) Macrophage depletion impairs wound healing and increases left ventricular remodeling after myocardial injury in mice. American Journal of Pathology, 170, 1093-1103. doi:10.2353/ajpath.2007.060547

[36] Okazaki, T., Ebihara, S., Asada, M., Yamanda, S., Saijo, Y., Shiraishi, Y., Ebihara, T., Niu, K., Mei, H., Arai, H. and Yambe, T. (2007) Macrophage colony-stimulating factor improves cardiac function after ischemic injury by inducing vascular endothelial growth factor production and survival of cardiomyocytes. American Journal of Pathology, 171, 1093-1103. doi:10.2353/ajpath.2007.061191 